\title{
VECTOR MEASURES WITH VARIATION IN A BANACH FUNCTION SPACE
}

\author{
O. BLASCO \\ Departamento de Análisis Matemático, \\ Universidad de Valencia, \\ Doctor Moliner, 50 \\ E-46100 Burjassot (Valencia), Spain \\ E-mail:Oscar.Blasco@uv.es \\ PABLO GREGORI \\ Departament de Matemàtiques, \\ Universitat Jaume I de Castelló, \\ Campus Riu Sec \\ E-12071 Castelló de la Plana (Castelló), Spain \\ E-mail:gregori@mat.uji.es
}

\begin{abstract}
Let $E$ be a Banach function space and $X$ be an arbitrary Banach space. Denote by $E(X)$ the Köthe-Bochner function space defined as the set of measurable functions $f: \Omega \rightarrow X$ such that the nonnegative functions $\|f\|_{X}: \Omega \rightarrow[0, \infty)$ are in the lattice $E$. The notion of $E$-variation of a measure - which allows to recover the $p$ variation (for $E=L^{p}$ ), $\Phi$-variation (for $E=L^{\Phi}$ ) and the general notion introduced by Gresky and Uhl - is introduced. The space of measures of bounded $E$-variation $V_{E}(X)$ is then studied. It is shown, among other things and with some restriction of absolute continuity of the norms, that $(E(X))^{*}=V_{E^{\prime}}\left(X^{*}\right)$, that $V_{E}(X)$ can be identified with space of cone absolutely summing operators from $E^{\prime}$ into $X$ and that $E(X)=V_{E}(X)$ if and only if $X$ has the RNP property.
\end{abstract}

\section{Introduction}

The concept of variation in the frame of vector measures has been fruitful in several areas of the functional analysis, such as the description of the duality of vector-valued function spaces such as certain Köthe-Bochner function spaces (Gretsky and $\mathrm{Uhl}^{10}$, Dinculeanu ${ }^{7}$ ), the reformulation of operator ideals such as the cone absolutely summing operators $\left(\right.$ Blasco $\left.^{4}\right)$, and the Hardy spaces of harmonic function $\left(\right.$ Blasco $\left.^{2,3}\right)$. The variation of a vector measure has been considered in more an more wide families of function spaces, starting with the $p$-variation in Dinculeanu ${ }^{7}$, and following with the $\Phi$-variation in $\mathrm{Uhl}^{16}$ or the $E$-variation in Gretsky and $\mathrm{Uhl}^{10}$ for certain 
Banach lattices $E$. Recently the authors have considered a new approach to $(p, q)$-variation in Blasco and Gregori ${ }^{5}$.

Here we present a theory of $E$-variation which covers the one in Gretsky and $\mathrm{Uhl}^{10}$, but also allows us to include more general Banach lattices.

We present in a second stage the development of the theory of Lorentz spaces $\Lambda$ and $M$ in the setting of vector measures. This will include as particular cases the spaces $V_{p, \infty}(X)$ and $V_{p, 1}(X)$ introduced in Blasco and Gregori ${ }^{5}$.

The research of this paper is placed into the theory of function spaces and vector measures, then some introductory notation and preliminary definitions are needed.

In order to fix notation, the underlying measure space $(\Omega, \Sigma, \mu)$ is an arbitrary finite nonatomic measure space. For arbitrary measurable set $A$, $D_{A}$ stands for the set of all finite partitions of $A$ into measurable subsets. Letter $X$ shall usually denote an arbitrary Banach space.

In reference to vector measure theory, we recall that a vector measure is a countably additive set function $F: \Sigma \rightarrow X$ for which the (total) variation is the measure $|F|: \Sigma \rightarrow[0,+\infty]$ defined by $|F|(A):=$ $\sup _{\pi \in D_{A}} \sum_{A_{i} \in \pi}\left\|F\left(A_{i}\right)\right\|_{X}$. The vector measure $F$ is said to be absolutely continuous with respect to $\mu$ (also called $\mu$-continuous and denoted by $F \ll \mu)$ when $\lim _{\mu(A) \rightarrow 0}\|F(A)\|_{X}=0$, and of bounded variation if the measure $|F|$ is finite, i.e. $|F|(\Omega)<+\infty$.

(Indefinite) Bochner integral of measurable functions provide $\mu$ continuous vector measures and of bounded variation. The converse, that is, if every $\mu$-continuous vector measure of bounded variation arises as the Bochner integral of a measurable function, is a geometric property that the Banach space $X$ might fulfill or not. It is called Radon-Nikodým Property and when satisfied it is denoted by $X \in(R N P)$ (see Diestel and $\mathrm{Uhl}^{6}$ ).

Further definitions of variation have led, since the 30's until the 70's, to the consideration of $p$-variation (Dinculeanu ${ }^{7}$ ), $\Phi$-variation ( $\mathrm{Uhl}^{16,17}$ ) and $E$-variation (Gretsky and $\mathrm{Uhl}^{10}$ ), with several applications to the study of Banach spaces and operator theory.

On the side of function spaces, we fix on the family of Banach function spaces (or Köthe function spaces, see Bennett and Sharpley ${ }^{1}$ for a complete reference).

In the set of nonnegative measurable functions on $\Omega$-actually equivalence classes of functions and denoted by $\mathcal{M}^{+}$-, equipped with the usual pointwise order, Banach function spaces can be expressed by means of a function norm $\rho$ satisfying, for $f, g \in \mathcal{M}^{+}$: 
(1) $\rho(f) \geq 0$ and $\rho(f)=0$ iff $f=0, \quad \rho(\alpha f)=\alpha \rho(f)$ for $\alpha \geq 0$ and $\rho(f+g) \leq \rho(f)+\rho(g)$.

(2) $f \leq g$ in $\mathcal{M}^{+}$implies $\rho(f) \leq \rho(g)$.

(3) $0 \leq f_{n} \uparrow f$ in $\mathcal{M}^{+}$implies $\rho\left(f_{n}\right) \uparrow \rho(f)$.

(4) $\mu(E)<\infty$ implies $\rho\left(\chi_{E}\right)<\infty$.

(5) $\mu(E)<\infty$ implies $\int_{E} f d \mu \leq C_{E} \rho(f)$.

The definition of $\rho$ is extended to $\mathcal{M}$ (the class of all scalar measurable functions on $\Omega$ ) by $\rho(f)=\rho(|f|)$ and ta Banach function space $E$ is given by the functions of $\mathcal{M}$ such that $\rho(f)<\infty$, taking the notation of $\|f\|_{E}$ for $\rho(f)$.

Since we are assuming $\mu(\Omega)<\infty$ from 4 and 5 we have that

$$
L^{\infty}(\mu) \subseteq E \subseteq L^{1}(\mu) .
$$

A "dual" norm is defined by $\|f\|^{\prime}=\sup \left\{\int_{\Omega}|f g| d \mu: g \in \mathcal{M}^{+}, \rho(g) \leq 1\right\}$.

It shares the properties of $\|\cdot\|_{E}$ (i.e., it is a function norm) and defines in $\mathcal{M}$ the called associate space $E^{\prime}$ by

$$
E^{\prime}=\left\{f \in \mathcal{M}:\|f\|_{E^{\prime}}:=\|f\|^{\prime}<\infty\right\} .
$$

The Hölder inequality reads in this case

$$
\int_{\Omega}|f g| d \mu \leq\|f\|_{E}\|g\|_{E^{\prime}}
$$

and the second "dual" norm (i.e., the dual norm of $\|\cdot\|^{\prime}$ ) is found to coincide with $\|\cdot\|_{E}$. In other words, $E^{\prime \prime}:=\left(E^{\prime}\right)^{\prime}=E$.

Two additional properties to be used latter on are the following:

Property $(J)$, i.e. if for every function $f \in E$ and every partition $\pi \in D_{\Omega}$ we have that

$$
\left\|\sum_{A \in \pi} \frac{\int_{A} f d \mu}{\mu(A)} \chi_{A}\right\|_{E} \leq\|f\|_{E} .
$$

$E^{\prime}$ has an absolutely continuous norm, i.e. if $\lim _{A \downarrow \emptyset}\left\|f \chi_{A}\right\|_{E^{\prime}}=0$ for all $f \in E^{\prime}$.

The notion of absolute continuity of the norm of $E$ is important since the topological dual space of $E, E^{*}$, is exactly the associate space $E^{\prime}$ whenever $E$ has absolutely continuous norm. The notation $E_{a}$ is used for the set of functions $f$ of $E$ with absolutely continuous norm, and $E$ is said to have absolutely continuous norm when $E=E_{a}$. Another subspace of $E$ is of great interest, $E_{b}$, the closure of the set of simple functions in $E$. The inclusion $\{0\} \subset E_{a} \subset E_{b} \subset E$ holds.

Note that if $E$ has property $(J)$ then also $E^{\prime}$ does, but this is not the case for $E$ having absolutely continuous norm (for instance $E=L^{1}$ ). 
Inside this wide family of scalar function spaces, there is an interesting class, playing a crucial role in the theory of interpolation of operators. It is the family of rearrangement invariant Banach function spaces.

For every measurable function $f$, the distribution function $\mu_{f}$ is given by

$$
\mu_{f}(\lambda)=\mu(\{w \in \Omega:|f(w)|>\lambda\}), \quad \lambda \geq 0 .
$$

Two measurable functions are said to be equimeasurable when their respective distribution functions coincide. The decreasing rearrangement of $f$ is the right-continuous inverse of $\mu_{f}$, that is,

$$
f^{*}(t)=\inf \left\{\lambda>0: \mu_{f}(\lambda) \leq t\right\}, \quad t \geq 0 .
$$

It is easy to see that $f$ and $f^{*}$ are always equimeasurable, and we have the Hardy-Littlewood inequality

$$
\int_{\Omega}|f g| d \mu \leq \int_{0}^{\infty} f^{*}(t) g^{*}(t) d t .
$$

The notion of resonant measure spaces arises at this point. However, as it is out of our scope, we just mention that these measure spaces are characterized by either nonatomicity, or if atomic, then completely atomic with all the atoms having the same measure. Keeping in mind that in our situation we have a finite nonatomic measure space then (see Bennett and Sharpley ${ }^{1}$ ) for every $t \in[0, \mu(\Omega)]$ there exists a measurable set $E_{t}$ so that

$$
\int_{E_{t}} f d \mu=\int_{0}^{t} f^{*}(s) d s .
$$

Hardy-Littlewood inequality motivates the definition of the maximal function of $f^{*}$

$$
f^{* *}(t)=\frac{1}{t} \int_{0}^{t} f^{*}(s) d s, \quad t>0,
$$

also called the second rearrangement of $f$.

A Banach function space $E$ is said rearrangement invariant when every two equimeasurable functions have always the same norm $\|\cdot\|_{E}$. In this case, the notion of fundamental function of $E$,

$$
\varphi_{E}(t)=\left\|\chi_{A}\right\|_{E}, \quad \mu(A)=t, \quad t \in[0, \mu(\Omega)]
$$

is of rather interest. It is a continuous (except perhaps at the origin) increasing function such that $\frac{\varphi_{E}(t)}{t}$ is decreasing and holding the relation

$$
\varphi_{E}(t) \varphi_{E^{\prime}}(t)=t, \quad t \in[0, \mu(\Omega)]
$$


Now, the notion of absolute continuity of the norm of $E$ (also seen as $\left.E_{a}=E\right)$ can be reformulated as $\varphi_{E}\left(0^{+}\right)=0$.

In a rearrangement invariant Banach function space $E$, one can take its fundamental function and define the Lorentz spaces $M(E)$ and $\Lambda(E)$, whose norms are given by

$$
\begin{aligned}
\|f\|_{M(E)} & =\sup _{0<t<\mu(\Omega)} f^{* *}(t) \varphi_{E}(t) \\
\|f\|_{\Lambda(E)} & =\int_{0}^{\mu(\Omega)} f^{*}(s) d \varphi_{E}(s) \\
& =\|f\|_{\infty} \varphi_{E}\left(0^{+}\right)+\int_{0}^{\mu(\Omega)} f^{*}(s) \phi_{E}(s) d s
\end{aligned}
$$

We have to mention that it is needed $\varphi_{E}$ to be concave, in order to have a good definition for $\Lambda(E)$, and that it is always possible after a convenient renorming in $E$. Therefore $\phi_{E}$ is well defined as the nonnegative decreasing function such that

$$
\varphi_{E}(t)=\varphi_{E}\left(0^{+}\right)+\int_{0}^{t} \phi_{E}(s) d s .
$$

Lorentz spaces $\Lambda(E)$ and $M(E)$ are rearrangement invariant spaces with fundamental function $\varphi_{E}$ (when it is chosen to be concave) and the inclusion $\Lambda(E) \subset E \subset M(E)$ is a norm-one embedding. The study of duality of those spaces lead to

$$
\Lambda(E)^{*}=M\left(E^{*}\right) \quad M(E)^{*}=\Lambda\left(E^{*}\right)
$$

when $\varphi_{E}\left(0^{+}\right)=0$.

Finally recall the vector-valued Banach function spaces, also called Köthe-Bochner function spaces. A Banach function space $E$ and an arbitrary Banach space $X$ give rise to the Köthe-Bochner function space $E(X)$, defined as the set of measurable functions $f: \Omega \rightarrow X$ such that the nonnegative functions $\|f\|_{X}: \Omega \rightarrow[0, \infty)$ are in the lattice $E$. Besides, $\|f\|_{E(X)}:=\|\| f\left\|_{X}\right\|_{E}$.

\section{Vector measures with variation in a Banach function space}

This section contains the definition of the vector space in the general case, some useful preliminary results, and the main theorems (concerning the Radon-Nikodým property, the compatibility with historical definitions see Dinculeanu ${ }^{7}, \mathrm{Uhl}^{16}$ and Gretsky and Uhl ${ }^{10}$-, the description of the 
dual of Köthe-Bochner function spaces and descriptions in terms of the space of cone absolutely summing operators).

Definition 2.1. Let $E$ be a Banach function space and $X$ an arbitrary Banach space, we define the space $V_{E}(\Omega, \Sigma, \mu, X)$ - denoted also as $V_{E}(X)$ as the space of all finitely additive vector measures $F: \Sigma \rightarrow X$ for which the supremum

$$
\sup \left\{\sum_{A \in \pi}\left|\alpha_{A}\right|\|F(A)\|: \quad \pi \in D_{\Omega},\left\|\sum_{A \in \pi} \alpha_{A} \chi_{A}\right\|_{E^{\prime}} \leq 1\right\}
$$

is bounded. This supremum is called the $E$-variation of $F$ and it is denoted by $|F|_{E}$.

Remark 2.1. The following basic properties are true:

(i) $\|F(A)\| \leq|F|_{E}\left\|\chi_{A}\right\|_{E^{\prime}}$ for all $A \in \Sigma$.

(ii) If $F \in V_{E}(X)$ then it has bounded variation.

(iii) If $E^{\prime}$ has absolutely continuous norm then $F \in V_{E}(X)$ implies $F \ll \mu$.

In particular if $F \in V_{E}(X)$ then $F$ is a countably additive vector measure.

(iv) If $E_{1} \subset E_{2}$ then $V_{E_{1}}(X) \subset V_{E_{2}}(X)$.

In particular $V_{L^{\infty}}(X) \subseteq V_{E}(X) \subseteq V_{L^{1}}(X)$.

Remark 2.2. A simple argument allows us to replace $\|F(A)\|$ by $|F|(A) \mid$ in the previous definition. $|F|_{E}=\sup \left\{\sum_{A \in \pi}\left|\alpha_{A}\right||F|(A)\right.$ : $\left.\left\|\sum_{A \in \pi} \alpha_{A} \chi_{A}\right\|_{E^{\prime}} \leq 1\right\}$.

Now we can give a characterization for vector measures of bounded $E$-variation in terms of the function space $E$.

Lemma 2.1. Let $E^{\prime}$ have norm absolutely continuous. The following assertions are equivalent:

(1) $F \in V_{E}(X)$,

(2) There exists $\varphi \geq 0, \varphi \in E$ such that

$$
|F|(A)=\int_{A} \varphi d \mu, \quad A \in \Sigma .
$$

Moreover $\|\varphi\|_{E}=|F|_{E}$.

Proof: For $F \in V_{E}(X)$, the set function $|F|$ is a $\mu$-continuous positive measure. The Radon-Nikodým theorem provides a nonnegative function 
$\varphi \in L^{1}$ representing $|F|$. Taking into account the duality of norms in $E$ and $E^{\prime}$

$$
\|\varphi\|_{E}=\sup \left\{\int_{\Omega} \varphi \psi d \mu: \quad\|\psi\|_{E^{\prime}} \leq 1\right\} .
$$

and approximating the supremum with the use of simple functions in $E^{\prime}$, we just have to replace $\varphi$ with $|F|$ to get that

$$
\|\varphi\|_{E}=\sup \left\{\sum_{A \in \pi}\left|\alpha_{A}\right||F|(A):\left\|\sum_{A \in \pi} \alpha_{A} \chi_{A}\right\|_{E^{\prime}} \leq 1\right\}=|F|_{E} .
$$

When $\varphi$ is as described in 2, the fact that $F \in V_{E}(X)$ is an immediate corollary of the Remark 2.2.

Proposition 2.1. $\left(V_{E}(X),|\cdot|_{E}\right)$ is a Banach space.

Proof: Norm properties of $|\cdot|_{E}$ are easy to check. For the completeness, let $\left\{F_{n}\right\}_{n=1}^{\infty} \subset V_{E}(X)$ be a Cauchy sequence. For every $A \in \Sigma$, the sequence $\left\{F_{n}(A)\right\}_{n=1}^{\infty} \subset X$ is also Cauchy (hence convergent) since

$$
\left\|F_{n}(A)-F_{m}(A)\right\|_{X} \leq\left\|\chi_{A}\right\|_{E^{\prime}}\left|F_{n}-F_{m}\right|_{E}, \quad m, n \in \mathbb{N}
$$

The set function $F$ defined as $F(A):=\lim _{n} F_{n}(A)$ for $A \in \Sigma$ is finitely additive.

Assuming $\left\{F_{n}\right\}$ not convergent in $V_{E}(X)$, we can find an $\varepsilon_{0}>0$ such that for every $k \in \mathbb{N}$ there is a $n_{k} \geq k$ and a simple function $s_{k}=\sum_{A \in \pi_{k}} \alpha_{A}^{k} \chi_{A}$ suct that $\left\|s_{k}\right\|_{E^{\prime}} \leq 1$ for which

$$
\varepsilon_{0}<\sum_{A \in \pi_{k}}\left|\alpha_{A}^{k}\right|\left\|F_{n_{k}}(A)-F(A)\right\|_{X} .
$$

Now let us take $k:=n\left(\frac{\varepsilon_{0}}{2}\right)$ (from the Cauchy's condition of $\left\{F_{n}\right\}_{n}$ with $\left.\frac{\varepsilon_{0}}{2}>0\right)$. Fix an integer $n_{k} \geq k$ and a simple function $s=\sum_{A \in \pi} \alpha_{A} \chi_{A}$ with $\|s\|_{E^{\prime}} \leq 1$. For any $m \geq n_{k}$ we have

$$
\begin{gathered}
\varepsilon_{0} \quad \sum_{A \in \pi}\left|\alpha_{A}\right|\left\|F_{n_{k}}(A)-F(A)\right\|_{X} \\
\leq \sum_{A \in \pi}\left|\alpha_{A}\right|\left\|F_{n_{k}}(A)-F_{m}(A)\right\|_{X}+\sum_{A \in \pi}\left|\alpha_{A}\right|\left\|F_{m}(A)-F(A)\right\|_{X} \\
\leq\left|F_{n_{k}}-F_{m}\right|_{E}+\sum_{A \in \pi}\left|\alpha_{A}\right|\left\|F_{m}(A)-F(A)\right\|_{X} \\
<\frac{\varepsilon_{0}}{2}+\sum_{A \in \pi}\left|\alpha_{A}\right|\left\|F_{m}(A)-F(A)\right\|_{X} .
\end{gathered}
$$

Therefore

$$
\sum_{A \in \pi}\left|\alpha_{A}\right|\left\|F_{m}(A)-F(A)\right\|_{X}>\frac{\varepsilon_{0}}{2}
$$

for every $m \geq k$, which leads to a contradiction.

Finally $F \in V_{E}(X)$ since $|F|_{E} \leq\left|F-F_{n}\right|_{E}+\left|F_{n}\right|_{E}<\infty$. 
Proposition 2.2. Let $E$ be a Banach function space satisfying property $(J)$. The space $V_{E}(X)$ can be reformulated with the equivalent expression of E-variation

$$
\sup _{\pi \in D_{\Omega}}\left\|\sum_{A \in \pi} \frac{F(A)}{\mu(A)} \chi_{A}\right\|_{E(X)}
$$

Proof: It is plain that for $\pi \in D_{\Omega}$ and the simple function $s=$ $\sum_{A \in \pi} \alpha_{A} \chi_{A}$, with $\|s\|_{E^{\prime}} \leq 1$, we have that

$$
\begin{gathered}
\sum_{A \in \pi}\left|\alpha_{A}\right|\|F(A)\|_{X}=\int_{\Omega}\left(\sum_{A \in \pi}\left|\alpha_{A}\right| \chi_{A}\right)\left(\sum_{A \in \pi} \frac{\|F(A)\|_{X}}{\mu(A)} \chi_{A}\right) d \mu \\
\leq\left\|\sum_{A \in \pi} \frac{\|F(A)\|_{X}}{\mu(A)} \chi_{A}\right\|_{E}
\end{gathered}
$$

which proves an inequality. The reverse inequality is obtained by means of the duality between norms in $E$ and $E^{\prime}$. Let us take arbitrary $\pi \in D_{\Omega}$. Then

$$
\begin{aligned}
\left\|\sum_{A \in \pi} \frac{\|F(A)\|_{X}}{\mu(A)} \chi_{A}\right\|_{E} & =\sup \left\{\int_{\Omega}\left(\sum_{A \in \pi} \frac{\|F(A)\|_{X}}{\mu(A)} \chi_{A}\right) g d \mu:\|g\|_{E^{\prime}} \leq 1\right\} \\
& =\sup \left\{\sum_{A \in \pi}\left(\frac{\int_{A} g d \mu}{\mu(A)}\right)\|F(A)\|_{X}:\|g\|_{E^{\prime}} \leq 1\right\} \\
= & \sup \left\{\sum_{A \in \pi}\left|\alpha_{A}\right|\|F(A)\|_{X}:\left\|\sum_{A \in \pi} \alpha_{A} \chi_{A}\right\|_{E^{\prime}} \leq 1\right\}
\end{aligned}
$$

where, for the last step, the inequality

$$
\left\|\sum_{A \in \pi}\left(\frac{\int_{A} g d \mu}{\mu(A)}\right) \chi_{A}\right\|_{E^{\prime}} \leq\|g\|_{E^{\prime}} \leq 1
$$

has been used, and was available since $E^{\prime} \in(J)$ from the hypothesis on $E$.

Proposition 2.3. Let $E$ be a Banach function space and let $X$ be a Banach space. Then $E(X) \subseteq V_{E}(X)$.

Proof: The operator $\lambda_{E}: E(X) \rightarrow V_{E}(X)$ given by the (indefinite) Bochner integral $f \mapsto \int_{(.)} f d \mu$ is the isometric inclusion, since for every (Bochner)-integrable function $f$, we have that $\left|\lambda_{E}(f)\right|(\cdot)=\int_{(\cdot)}\|f\|_{X} d \mu$. Assuming $\|f\|_{X} \in E$, we conclude that $\lambda_{E}(f) \in V_{E}(X)$ and $\left|\lambda_{E}(f)\right|_{E}=$ \|\|$f\left\|_{X}\right\|_{E}=\|f\|_{E(X)}$.

Theorem 2.1. Let $E^{\prime}$ have absolutely continuous norm and let $X$ be a Banach space. Then $E(X)=V_{E}(X)$ if and only if $X \in(R N P)$. 
Proof: Assume that $X \in(R N P)$. As long as any vector measure $F \in$ $V_{E}(X)$ is $\mu$-continuous and of bounded total variation, our assumption provides a function $f \in L^{1}(X)$ such that $F(A)=\int_{A} f d \mu$. Since $|F|(\cdot)=$ $\int_{(\cdot)}\|f\|_{X} d \mu$ it follows that $f \in E(X)$.

For the converse assume that $\lambda_{E}: E(X) \rightarrow V_{E}(X)$ given by $f \mapsto$ $\int_{(\cdot)} f d \mu$ is surjective. We shall prove that every bounded linear operator $T: L^{1} \rightarrow X$ is representable by some function $g \in L^{\infty}(X)$ (characterizing therefore the $(R N P)$ for $X)$.

For this purpose we shall deal with the Lebesgue measure space $([0,1], m)$. Let $T: L^{1} \rightarrow X$ be bounded and linear operator. Let us define the measure $G: \Sigma \rightarrow X$ by $G(A):=T\left(\chi_{A}\right)$ for every measurable set $A$. Since $\|G(A)\|_{X} \leq\|T\| m(A)$. This shows that $G \in V^{\infty}(X)$, i.e. $\|G\|_{V^{\infty}(X)}=\sup _{A} \frac{\|G(A)\|_{X}}{m(A)} \leq\|T\|<\infty$. Since $V^{\infty}(X)=V_{L^{\infty}}(X) \subset$ $V_{E}(X)$ and the surjection $V_{E}(X)=E(X)$ we have a function $g \in E(X) \subset$ $L^{1}(X)$ such that $G(A)=\int_{A} g d \mu$ for any measurable set $A$. The Lebesgue Differentiation Theorem yields

$$
\|g(t)\|_{X}=\left\|\lim _{h \rightarrow 0} \frac{1}{h} \int_{t}^{t+h} g(s) d s\right\|_{X}=\lim _{h \rightarrow 0} \frac{\|G([t, t+h])\|_{X}}{h} \leq\|T\|
$$

for almost all $t \in[0,1]$. Therefore $g \in L^{\infty}(X)$ and $T(\varphi)=\int_{\Omega} \varphi g d \mu$ for all $\varphi \in L^{1}$. Hence $X \in(R N P)$.

Vector measures and linear operators defined on the set of simple functions have always been connected by $F_{T}(A)=T\left(\chi_{A}\right)$ and $T_{F}\left(\sum_{A \in \pi} \alpha_{A} \chi_{A}\right)=\sum_{A \in \pi} \alpha_{A} F(A)$. The space of all linear bounded operators from a Banach function space $E$ to an arbitrary Banach space $X$ can be found isometric (or only isomorphic) to a space of vector measures with weak $E$-variation (see Gretsky ${ }^{9}$, Gretsky and $\mathrm{Uhl}^{10}$ and Gregori ${ }^{8}$ ). Now that we are restricted to the space $V_{E}(X)$, we show the ideal operator which is in correspondence with this vector measure space: the cone absolutely summing operators.

Definition 2.2. (see Schaefer ${ }^{14}$ ) Let $E$ be a Banach lattice and $B$ a Banach space. A linear operator from $E$ to $B$ is said to be cone absolutely summing (c.a.s.) if there is a constant $C>0$ such that for every $k \in \mathbb{N}$ and every family $e_{1}, e_{2}, \ldots, e_{k} \in E$ of positive elements we have

$$
\sum_{i=1}^{k}\left\|T\left(e_{k}\right)\right\|_{B} \leq C \sup _{\left\|e^{*}\right\|_{E^{*}} \leq 1} \sum_{i=1}^{k}\left|\left\langle e_{i}, e^{*}\right\rangle\right| .
$$

Denoting by $\Lambda^{1}(E, B)$ the set of such operators, its norm is given by the infimum of such constants $C$ holding the previous inequality and it denoted 
by $\|T\|_{\text {c.a.s. }}$.

Remark 2.3. If $E$ is a Banach function space and $e_{1}, e_{2}, \ldots, e_{k}$ positive elements then

$$
\sup _{\left\|e^{*}\right\|_{E^{*}} \leq 1} \sum_{i=1}^{k}\left|\left\langle e_{i}, e^{*}\right\rangle\right|=\left\|\sum_{i=1}^{k} e_{i}\right\|_{E} .
$$

Proposition 2.4. (see Schaefer $\left.{ }^{14}\right) T \in \Lambda^{1}(E, X)$ if and only if there exists $\phi_{T} \in E^{\prime}, \phi_{T} \geq 0$ such that

$$
\|T(f)\| \leq \int_{\Omega}|f| \phi_{T} d \mu .
$$

Moreover $\|T\|_{\text {c.a.s. }}=\left\|\phi_{T}\right\|_{E^{\prime}}$.

Theorem 2.2. Let $E$ be a Banach function space with absolutely continuous norm and $X$ a Banach space. Then $\Lambda^{1}(E, X)=V_{E^{\prime}}(X)$

Proof: If $T \in \Lambda^{1}(E, X)$, the measure $F_{T}: \Sigma \rightarrow X$ associated to $T$ belongs to $V_{E^{\prime}}(X)$, because if $A \in \Sigma$ then Proposition 2.4 gives $\left\|F_{T}(A)\right\| \leq$ $\int_{A} \phi_{T} d \mu$ for all $A \in \Sigma$ and some $\phi_{T} \in E^{\prime}$ and then

$$
\begin{aligned}
\sup \left\{\sum_{A \in \pi}\left|\alpha_{A}\right|\|F(A)\|\right. & \left.:\left\|\sum_{A \in \pi} \alpha_{A} \chi_{A}\right\|_{E} \leq 1\right\} \\
& \leq \sup \left\{\int_{\Omega}\left(\sum_{A \in \pi}\left|\alpha_{A}\right| \chi_{A}\right) \phi_{T} d \mu:\left\|\sum_{A \in \pi} \alpha_{A} \chi_{A}\right\|_{E} \leq 1\right\} \\
& =\left\|\phi_{T}\right\|_{E^{\prime}}=\|T\|_{\text {c.a.s. }}
\end{aligned}
$$

Conversely, since the norm in $\Lambda^{1}(E, X)$ coincides with

$$
\|T\|_{\text {c.a.s. }}=\sup \left\{\sum_{i=1}^{n}\left\|T \varphi_{i}\right\|_{X}: n \in \mathbb{N},\left\|\sum_{i=1}^{n} \varphi_{i}\right\|_{E} \leq 1, \varphi_{1}, \ldots, \varphi_{n} \geq 0\right\}
$$

Applying Lemma 2.1 we have that, for every function $f$ simple function,

$$
\left\|T_{F}(f)\right\|_{X} \leq \int_{\Omega}|f| \varphi d \mu
$$

where $\varphi$ represents $|F|$.

Therefore, for $\varphi_{1}, \ldots, \varphi_{N} \geq 0, \varphi_{i} \in E_{b}=E$ we have

$$
\sum_{n=1}^{N}\left\|T_{F}\left(\varphi_{n}\right)\right\|_{X} \leq \sum_{n=1}^{N} \int_{\Omega} \varphi_{n} \varphi d \mu=\int_{\Omega}\left(\sum_{n=1}^{N} \varphi_{n}\right) \varphi d \mu \leq|F|_{E^{\prime}}\left\|\sum_{n=1}^{N} \varphi_{n}\right\|_{E} .
$$

and then $\left\|T_{F}\right\|_{\text {c.a.s. }} \leq\|F\|_{E^{\prime}}$. 
Theorem 2.3. Let $X$ be a Banach space and $E$ be a Banach function space with absolutely continuous norm. Then $V_{E^{\prime}}\left(X^{*}\right)=(E(X))^{*}$.

Proof: Let $F \in V_{E^{\prime}}\left(X^{*}\right)$. Define $\Phi_{F}: E(X) \rightarrow \mathbb{K}$ using

$$
\Phi_{F}\left(\sum_{n=1}^{N} x_{n} \chi_{A_{n}}\right)=\sum_{n=1}^{N}\left\langle F\left(A_{n}\right), x_{n}\right\rangle .
$$

Recall that simple functions are dense in $E(X)$ because of the assumption and let $s=\sum_{n=1}^{N} x_{n} \chi_{A_{n}}$ be a simple function in $E(X)$,

$$
\left|\Phi_{F}(s)\right|=\left|\sum_{n=1}^{N}\left\langle F\left(A_{n}\right), x_{n}\right\rangle\right| \leq \sum_{n=1}^{N}\left\|x_{n}\right\|_{X}|F|\left(A_{n}\right)=\int_{\Omega}\|s(\cdot)\|_{X} \varphi d \mu
$$

where the function $\varphi$ is the one of Lemma 2.1. Applying now Hölder's inequality and density of simple functions, we get $\left\|\Phi_{F}\right\| \leq\|\varphi\|_{E^{\prime}}=|F|_{E^{\prime}}$.

In order to see the surjectivity, let us consider the measure $F_{\Phi}: \Sigma \rightarrow X^{*}$ induced by an element $\Phi$ of $(E(X))^{*}$. Let $\left\|\sum_{a \in \pi} \alpha_{A} \chi_{a}\right\|_{E} \leq 1$,

$$
\begin{array}{r}
\sum_{A \in \pi}\left|\alpha_{A}\|\| F_{\Phi}(A) \|_{X^{*}} \quad \sup _{\left\|x_{A}\right\| \leq 1}\right| \sum_{A \in \pi} \Phi\left(\alpha_{A} x_{A} \chi_{A}\right) \mid \\
=\sup _{\left\|x_{A}\right\| \leq 1}\left|\Phi\left(\sum_{A \in \pi} \alpha_{A} x_{A} \chi_{A}\right)\right| \\
\leq\|\Phi\| \sup _{\left\|x_{A}\right\| \leq 1}\left\|\sum_{A \in \pi} \alpha_{A} x_{A} \chi_{A}\right\|_{E(X)} \\
=\|\Phi\|
\end{array}
$$

Then $F_{\Phi} \in V_{E^{\prime}}\left(X^{*}\right)$ and also the isometry is proved.

\section{Variation in rearrangement invariant function spaces}

Rearrangement invariant Banach function spaces (r.i. spaces for short) satisfy property $(J)$ (see Bennett and Sharpley ${ }^{1}$ ). This family of spaces is very important in the theory of interpolation of operators. A part of the theory of rearrangement invariant function spaces - the one concerning the Lorentz spaces $\Lambda(E)$ an $M(E)$ associated to every rearrangement invariant function space $E$ - is going to be transferred to the setting of vector measures.

Definition 3.1. (see Blasco and Gregori $\left.{ }^{5}\right)$ Let $(\Omega, \Sigma, \mu)$ be a measure space and let us denote the interval $I=(0, \mu(\Omega)]$. Let $X$ be an arbitrary Banach 
space. For every finitely additive set function $F: \Sigma \rightarrow X$ we define the function

$$
\omega_{F}(t)=\sup _{\mu(A) \leq t}|F|(A), t \in I
$$

Proposition 3.1. (see Blasco and Gregori ${ }^{5}$ ) For an arbitrary vector mesure $F$, the following properties are true:

(1) $\omega_{F}$ is nondecreasing and subadditive.

(2) If $\mu$ is resonant then the function $\omega_{F}$ is continuous and concave.

(3) Let $F$ be of finite total variation. Then $F \ll \mu$ iff $w\left(0^{+}\right)=0$

(4) If $F=f d \mu$ for some $f \in L^{1}(\mu)$ then $\omega_{F}(t)=t f^{* *}(t)$ for all $t \in I$.

Now we are presenting a bigger measure space for $V_{E}(X)$, in a similar way as the Lebesgue-Marcinkiewicz $L^{p, \infty}$-also referred as weak- $L^{p}$ contains $L^{p}$.

Definition 3.2. The space $V_{E, \infty}(\Omega, \Sigma, \mu)$ - denoted also as $V_{E, \infty}(X)$ - is the space of all finitely additive vector measures $F: \Sigma \rightarrow X$ for which

$$
|F|(A) \leq C\left\|\chi_{A}\right\|_{E^{\prime}}
$$

for every mesurable set $A$ and some constant $C>0$ independent of $A$. The infimum of those constants $C$ is the norm, denoted by $|F|_{E, \infty}$.

Remark 3.1. (i) If $E$ is a r.i. space then $V_{E, \infty}(X)$ is given by all finitely additive vector measures $F$ of bounded variation such that

$$
\omega_{F}(t) \leq C \varphi_{E^{\prime}}(t) \forall t \in I .
$$

(ii) If $E^{\prime}$ has absolutely continuous norm then $F \in V_{E, \infty}(X)$ implies $F \ll \mu$ and $F$ is countably additive.

Definition 3.3. The space $\mathcal{V}_{E, \infty}(\Omega, \Sigma, \mu)$ - denoted also as $\mathcal{V}_{E, \infty}(X)$ - is the space of all finitely additive vector measures $F: \Sigma \rightarrow X$ for which

$$
\|F(A)\| \leq C\left\|\chi_{A}\right\|_{E^{\prime}}
$$

for every mesurable set $A$ and some constant $C>0$ independent of $A$. The infimum of those constants $C$ is the norm, denoted by $\|F\|_{E, \infty}$.

Theorem 3.1. Let $E$ be a r.i. space such that $E$ has norm absolutely continuous. Then $\mathcal{V}_{E^{\prime}, \infty}(X)=L(\Lambda(E), X)$.

Proof: Clearly if $T \in L(\Lambda(E), X)$ then $F_{T}(A)=T\left(\chi_{A}\right)$ defines a finitely additive measure in $\mathcal{V}_{E^{\prime}, \infty}(X)$ and $\left\|F_{T}\right\|_{E, \infty} \leq\|T\|$. 
Conversely, given $F \in \mathcal{V}_{E^{\prime}, \infty}(X)$ and defining $T_{F}$ in the natural way, we have that if $f$ is a positive simple function $f=\sum_{i=1}^{n} \alpha_{i} \chi_{A_{i}}$ where $A_{1} \subseteq A_{2} \ldots \subseteq A_{n}$ then we can write

$$
\begin{aligned}
\left\|T_{F}\left(\sum_{i=1}^{n} \alpha_{i} \chi_{A_{i}}\right)\right\| & =\left\|\sum_{i=1}^{n} \alpha_{i} F\left(A_{i}\right)\right\| \leq \sum_{i=1}^{n} \alpha_{i}\left\|F\left(A_{i}\right)\right\| \\
& \leq C \sum_{i=1}^{n} \alpha_{i} \varphi_{E}\left(\mu\left(A_{i}\right)\right)=C \int_{0}^{\mu(\Omega)} \sum_{i=1}^{n} \alpha_{i} \chi_{\left(0, \mu\left(A_{i}\right)\right)}(s) d \varphi_{E}(s) \\
& =C \int_{0}^{\mu(\Omega)} f^{*}(s) d \varphi_{E}(s)=C\|f\|_{\Lambda(E)} .
\end{aligned}
$$

For a general real-valued simple function write $f=f^{+}-f^{-}$where $f^{+}, f^{-}$are non-negative simple functions and $\left\|f^{+}\right\|_{\Lambda(E)},\left\|f^{-}\right\|_{\Lambda(E)} \leq$ $\|f\|_{\Lambda(E)}$. Since $T_{F}(f)=T_{F}\left(f^{+}\right)-T_{F}\left(f^{-}\right)$we get $\left\|T_{F}(f)\right\| \leq 2\|f\|_{\Lambda(E)}$.

Now a density argument finish the result because simple functions are dense in $\Lambda(E)$ due to the absolutely continuous norm of $E$.

The Lebesgue-Marcinkiewicz space $L^{p, \infty}$ is nothing but the Lorentz space $M\left(L^{p}\right)$. The following result shows that these new vector measure spaces are very related to the ones defined in the precedent section with the additional ingredient of Lorentz spaces.

Theorem 3.2. Let $E$ be a r.i. space such that $E^{\prime}$ has norm absolutely continuous and $X$ a Banach space. Then $V_{E, \infty}(X)=V_{M(E)}(X)$.

Proof: From Remark 2.2 one gets $|F|(A) \leq|F|_{M(E)}\left\|\chi_{A}\right\|_{E^{\prime}}$. Hence the inclusion $V_{M(E)}(X) \subseteq V_{E, \infty}(X)$ holds.

Conversely if $F \in V_{E, \infty}(X)$ then, using the Radon-Nikodym theorem, we get $\phi \in L^{1}(\mu)$ such that $|F|(A)=\int_{A} \phi d \mu$ for all $A \in \Sigma$. From 4 in Propostion 3.1 we get that $w_{F}(t)=t \phi^{* *}(t)$. Hence $\phi \in M(E)$, because $\varphi_{E}(t) \phi^{* *}(t)=\frac{\varphi_{E}(t)}{t} w_{F}(t)=\leq \frac{1}{\varphi_{E^{\prime}}(t)} w_{F}(t) \leq C$. This shows the corresponding isometry.

Part 2 in the Proposition 3.1 allows us to give the following definition:

Definition 3.4. In a resonant measure space $(\Omega, \Sigma, \mu)$ where the function $\omega_{F}$ is defined for every vector measure $F$, we define the function $W_{F}$ on the interval $I$ as the nonnegative, decreasing function such that for $t \in I$

$$
\omega_{F}(t)=\omega_{F}\left(0^{+}\right)+\int_{0}^{t} \mathrm{~W}_{F}(s) d s .
$$

Now we are introducing certain space contained in $V_{E}(X)$, in a similar way as the Lorentz space $L^{p, 1}$ is contained in $L^{p}$. 
Definition 3.5. The space $V_{E, 1}(\Omega, \Sigma, \mu)$-denoted also as $V_{E, 1}(X)$ - is the space of all $\mu$-continuous vector measures $F: \Sigma \rightarrow X$ for which

$$
|F|_{E, 1}=\int_{0}^{\mu(\Omega)} \mathrm{W}_{F}(s) d \varphi_{E}(s)<\infty
$$

Theorem 3.3. Let $E$ be a r.i. space and $X$ a Banach space. Then $V_{E, 1}(X)=V_{\Lambda(E)}(X)$.

Proof: If $F$ is $\mu$-continuous and of bounded variation then we have $\omega_{F}(t)=\int_{0}^{t} \mathrm{~W}_{F}(s) d s$. Assuming that $\phi$ represents the variation of $|F|$ then $\int_{0}^{t} \mathrm{~W}_{F}(s) d s=\int_{0}^{t} \phi^{*}(s) d s$ for all $t>0$. Therefore $\phi^{*}=\mathrm{W}_{F}$. Hence $\phi \in \Lambda(E)$ (or equivalently $F \in V_{\Lambda(E)}(X)$ ) if and only if $F \in V_{E, 1}(X)$.

\section{Acknowledgments}

The authors have been partially supported by grant DGESIC PB98-0146.

\section{References}

1. C. Bennett, R. Sharpley, Interpolation of operators, Pure and applied mathematics vol. 129, Academic Press, Inc., New York (1988).

2. O. Blasco, Boundary values of functions in vector-valued Hardy spaces and Geometry of Banach spaces, J. Funct. Anal., Vol 78 (1988) 346-364.

3. O. Blasco, Boundary values of vector valued functions in Orlicz-Hardy classes, Arch. Math., Vol. 49 (1987) 434-439.

4. O. Blasco, Positive $p$-summing operators, vector measures and tensor products, Proc. Edinburgh Math. Soc. 31 (1988), n. 2, 179-184.

5. O. Blasco, P. Gregori. Lorentz spaces of vector measures, (2002) (to appear).

6. J. Diestel, J.J. Uhl, Vector measures, American Mathematical Society, Mathematical Surveys, Number 15, R. I. (1977).

7. N. Dinculeanu, Vector measures, International Series of Monographs in Pure and Applied Mathematics, Volume 95, Pergamon Press (1967).

8. P. Gregori, Espacios de medidas vectoriales, PhD Thesis, Valencia (2001).

9. N.E. Gretsky, Representation theorems on Banach function spaces, Trans. Amer. Math. Soc., Vol 167 (1972), 263-277.

10. N.E. Gretsky, J.J. Uhl, Bounded linear operators on Banach function spaces of vector-valued functions, Trans. Amer. Math. Soc., Vol 167 (1972), 263-277.

11. P.R. Halmos, Measure theory, Van Nostrand, New York (1950).

12. J. Lindenstrauss, L. Tzafriri, Classical Banach spaces II. Function spaces, Springer-Verlag, Berlin, (1979).

13. G.G. Lorentz, Some new functional spaces, Ann. Math., Vol 51 (1950), $37-55$. 
14. H.H. Schaefer, Banach lattices and positive operators, Springer-Verlag, New York (1974).

15. E.M. Stein, G. Weiss, Introduction to Fourier analysis on euclidean spaces, Princeton University Press, Princeton (1971).

16. J.J. Uhl, Orlicz spaces of finitely additive set functions, Studia Mathematica T. XXIX (1967) 19-58

17. J. J. Uhl, Orlicz spaces of finitely additive set functions, linear operators, and martingales, Bull. Amer. Math. Soc. 73 (1967) 116-119.

18. J. J. Uhl, On a class of operators on Orlicz spaces, Studia Math. 40 (1971) $17-22$. 